\title{
Primary intrarenal neuroblastoma in a 13-month-old girl presenting as a Wilms' tumor with pulmonary metastasis
}

\author{
Onur Telli ${ }^{1}$, Ufuk Ateş ${ }^{2}$, Ergun Ergun ${ }^{2}$, Rahşan Vargun Yıldız ${ }^{3}$, Banu Yağmurlu ${ }^{4}$, Bilgehan \\ Yalçın5, Banu Bilezikçí6, Gülnur Göllü², Aydın Yağmurlu² \\ Departments of ${ }^{1}$ Pediatric Urology, and ${ }^{2}$ Pediatric Surgery, Ankara University, Faculty of Medicine; Clinics of ${ }^{3}$ Pediatric \\ Surgery and ${ }^{6}$ Pathology Ankara Güven Hospital; ${ }^{4}$ Radiology Clinic, Ankara Memorial Hospital; ${ }^{5}$ Division of Pediatric \\ Oncology, Department of Pediatrics, Hacettepe University Faculty of Medicine, Ankara, Turkey. \\ E-mail: drergunergun@gmail.com
}

Received: 1st March 2016, Revised: 5th April 2016, Accepted: 25th May 2016

\begin{abstract}
SUMMARY: Telli O, Ateş U, Ergun E, Vargun-Yıldız R, Yağmurlu B, Yalçın B, Bilezikçi B, Göllüi G, Yağmurlu A. Primary intrarenal neuroblastoma in a 13-month-old girl presenting as a Wilms' tumor with pulmonary metastasis. Turk J Pediatr 2016; 58: 532-534.

We report a 13-month-old girl with primary intrarenal neuroblastoma initially diagnosed as Wilms' tumor. Intrarenal neuroblastoma is exceedingly rare in pediatric age that may masquerade as Wilms' tumor clinically and radiographically and it is important to differentiate for management.
\end{abstract}

Key words: intrarenal neuroblastoma, Wilms' tumor, pediatric.

Primary intrarenal neuroblastoma is a rare condition and pulmonary metastases of neuroblastoma are extremely rare. It may also resemble Wilms tumor that may cause diagnostic dilemma and inadequate management. Intrarenal neuroblastoma is believed to originate from adrenal rests found within renal tissue or from intrarenal sympathetic ganglia. Clinically and radiologically intrarenal neuroblastoma mimics Wilms' tumor, and as primary intrarenal neuroblastoma has a much poorer prognosis than Wilms' tumor and requires more aggressive neoadjuvant chemotherapy and close postoperative surveillance due to the likelihood of recurrence. ${ }^{1}$

\section{Case Report}

A 13-month-old girl presented with left-sided abdominal mass, identified by her mother. Physical examination revealed a unilateral, palpable, firm, abdominal mass extending to more than $10 \mathrm{~cm}$ below the left costal margin. The patient had no urinary or bowel complaints. Routine laboratory data were within normal limits. Urine revealed normal levels of vanillylmandelic (VMA) and homovanillic acids (HVA). Computed tomography (CT) scan of thorax and abdomen was performed, which showed an extensive tumoral lesion, size of $140 \times 66 \times 90 \mathrm{~mm}$ in left kidney with dilatation of calyceal system, some deformity, and some scattered calcification and multiple bilateral lung nodules consistent with metastatic disease (Fig. 1). Left radical nephrectomy was performed with enlarged periaortic lymph excision. Pathologic study revealed a poorly differentiated intrarenal neuroblastoma affecting $80 \%$ of the kidney with vascular and lymphatic invasion but no adrenal involvement and foci of necrosis were found. Immunohistochemistry revealed NSE, chromogranin, synaptophysin positive (Fig. 2 ). The distinction of this rare tumor from Wilms' tumor presents an important challenge since both tumors have major differences in prognostic and therapeutic response.

\section{Discussion}

As the management and the outcome of intrarenal neuroblastoma and Wilms tumor are very different, both are common intraabdominal malignancies of early childhood and it is important to differentiate these malignancies. Wilms' tumor is the most common solid intrarenal neoplasm of childhood, with a reported incidence of about 8.3 cases per million in children younger than fifteen years ${ }^{2}$. However, very rarely intrarenal neuroblastoma 


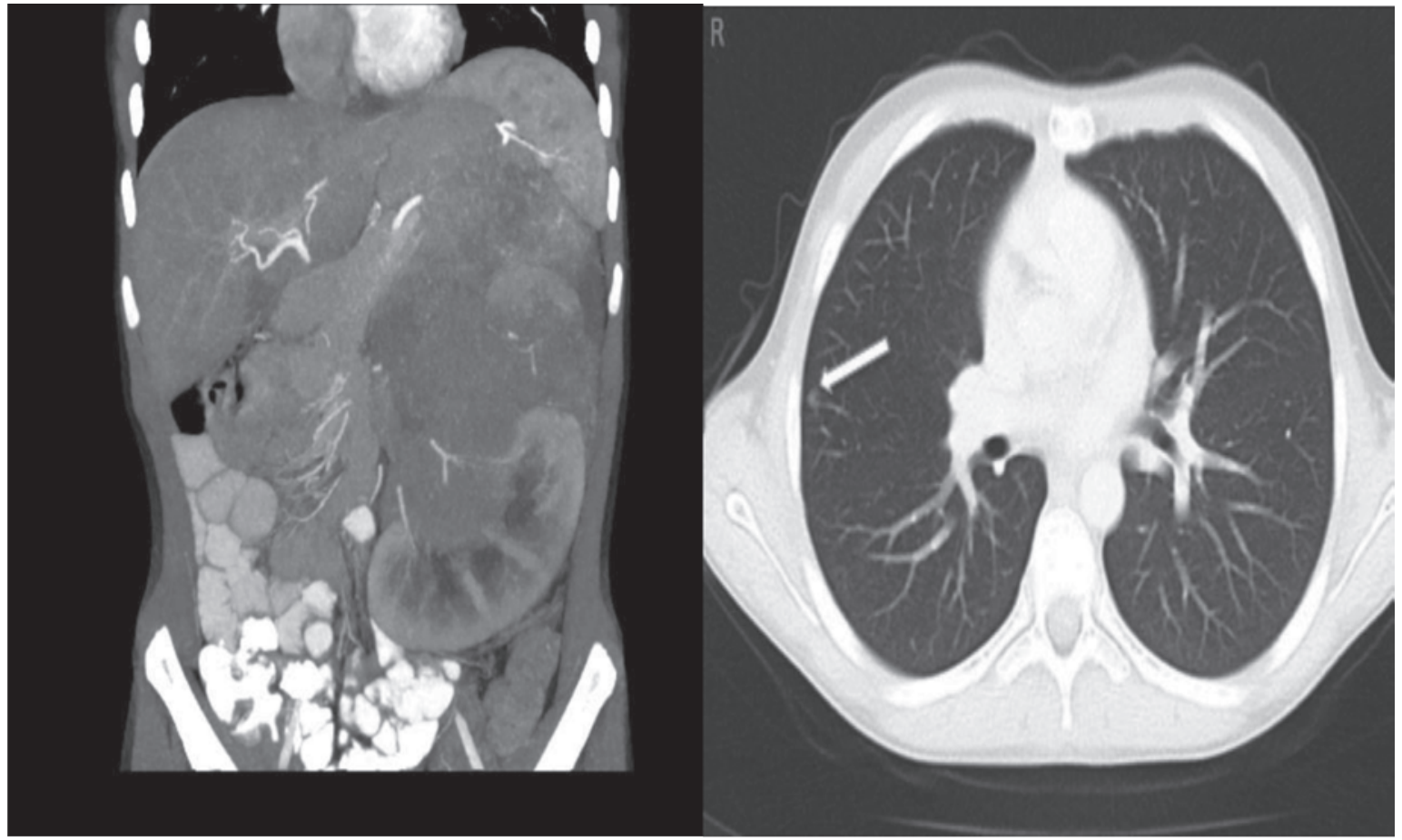

Fig. 1. CT scan of abdomen and chest; A) Coronal image of large abdominal mass in left side B) Axial image of the chest displays one of the multiple solid pulmonary nodules (arrow) which were thought to represent Wilms tumor metastases

may develop in children. Neuroblastoma has been reported that it can originate from adrenal rests in an abnormal location or from intrarenal sympathetic ganglia ${ }^{3,4}$. Clinical, radiological, and pathological correlation is very essential for diagnosis and appropriate management of this type of unusual cases. Similar to our case, reported cases of intrarenal neuroblastoma where the diagnosis was clinically suspected and diagnosed only after kidney removal, the misdiagnosis might affect therapy in several ways ${ }^{5}$. Some authors report guided fine needle aspiration is a very accurate and useful technique for preoperative diagnosis of renal masses ${ }^{6}$.

Hypertension in a child with renal tumor is highly suspicious of neuroblastoma. However, in our case the patient was not hypertensive. It was reported that $27 \%$ for neuroblastoma and a higher incidence of hypertension (66-100\%) has been associated with intrarenal neuroblastoma. Moreover, urinary catecholamines could be negative or high in this group of patients ${ }^{7}$.

Some radiological features of suprarenal neuroblastoma may not be seen with intrarenal neuroblastoma that may present as Wilms tumor. Those are suprarenal location, speckled calcification, multifocal, mottled, or ring-like calcification, anterior displacement of the great vessels by paraaortic and paracaval adenopathy and renal displacement by hilar disease ${ }^{8}$. In our case and in almost all cases reported in the literature renal neuroblastomas do not have the characteristic calcification, and this feature has been reported previously ${ }^{9}$. Lung metastases in neuroblastoma at presentation are very rare $(3.6 \%)$ and are more commonly seen in patients with MYCN oncogene amplified tumors, those with elevated lactate dehydrogenase levels, or with an adrenal primary ${ }^{10}$.

In our case, after the diagnosis of neuroblastoma was confirmed, further studies were performed. Meta-iodobenzyl-guanidine scan was negative, and bone marrow aspirate was normal. The girl was diagnosed as a stage IV neuroblastoma with unfavorable histology (Shimada classification) and received further adjuvant therapy according to the NB 99 protocol followed by a stem cell transplant. The patient remains under close surveillance without relapse after 6-month follow-up CT; showed further reduction in pulmonary metastases and no evidence of residual or recurrent abdominal tumor. 

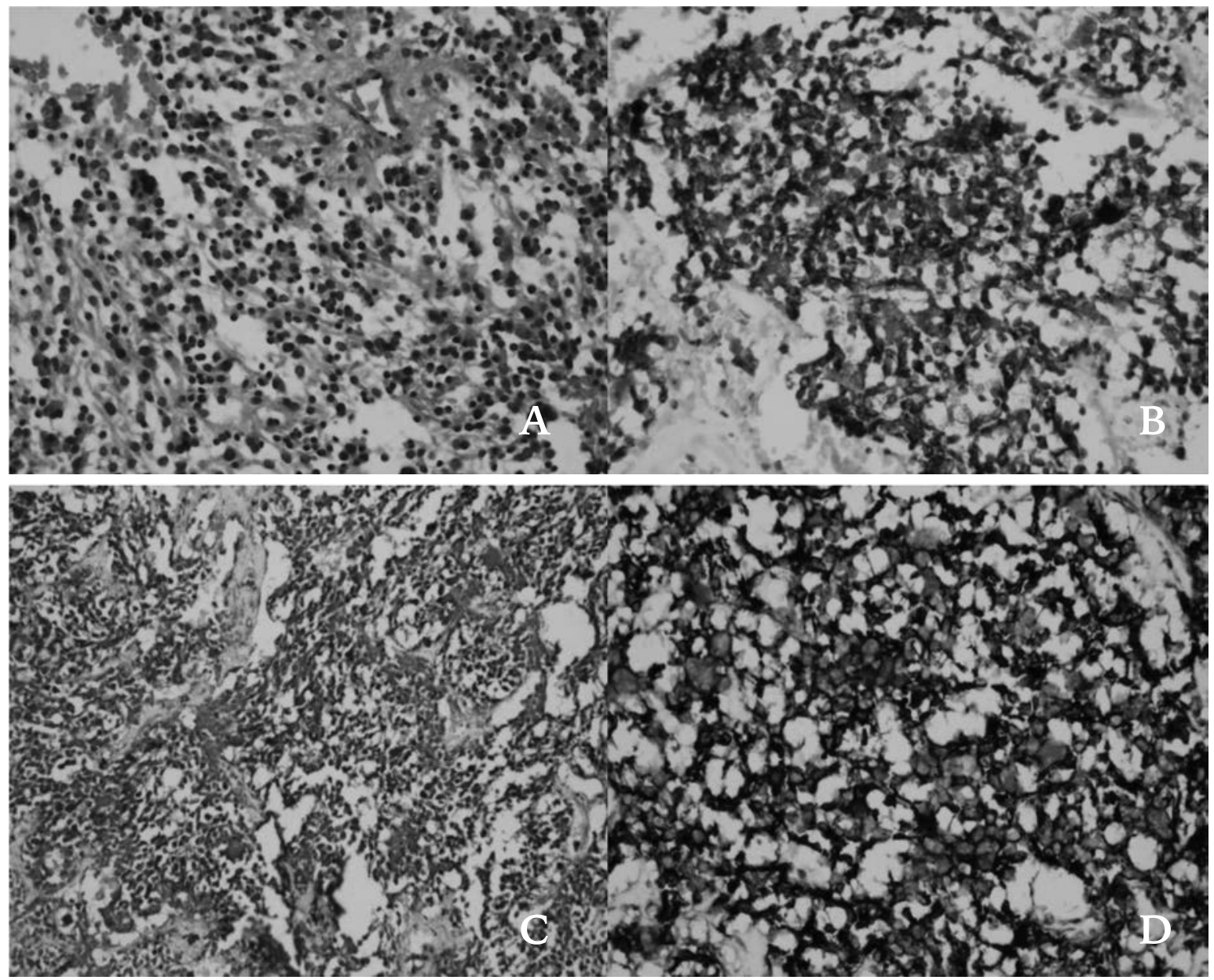

Fig. 2. Histological findings of the neuroblastoma A) The tumor cells are small and regular, with round, deeply staining nuclei, and little cytoplasms (HE, X40) B) Immunohistochemically, neuroblastoma cells express synaptophysin, x40 C) Immunohistochemically, neuroblastoma cells express NSE, x20 D) Immunohistochemically, neuroblastoma cells express CD56, $\mathrm{x} 40$

In conclusion, intrarenal neuroblastoma may radiologically and clinically present as Wilms' tumor. Distinction of this tumor from Wilms tumor is crucial since both tumors have different prognostic and therapeutic responses.

\section{REFERENCES}

1. Sellaturay SV, Arya M, Banisadr S, Murthi GV, Sebire NJ, Duffy PG. Primary intrarenal neuroblastoma: a rare, aggressive tumour of childhood mimicking Wilms' tumour. J Pediatr Urol 2006; 2: 522-524.

2. Kessler OJ, Siegel JF, Brock WA. Intrarenal neuroblastoma masquerading as Wilms' tumor. Urology 1998; 51: 313316.

3. Schechter DC. Aberrant adrenal tissue. Ann Surg 1968; 167: 421-426.

4. Tiniakos D, Anagnostou V, Stavrakis S, Karandrea D, Agapitos E, Kittas C. Ontogeny of intrinsic innervation in the human kidney. Anat Embryo 2004; 209: 41-47.
5. Dickson PV, Sims TL, Streck CJ, et al. Avoiding misdiagnosing neuroblastoma as Wilms tumor. J PediatrSurg 2008; 43: 1159-1163.

6. Serrano R, Rodríguez-Peralto JL, De Orbe GG, Melero C, de Agustín P. Intrarenal neuroblastoma diagnosed by fine-needle aspiration: a report of two cases. Diagn Cytopathol 2002; 27: 294-297.

7. Lall A, Bajpai M, Gupta DK. Intrarenal neuroblastoma-a diagnostic dilemma: A report of three cases. Indian J Urol 2001; 17: 170-172.

8. Peretz GS, Lam AH. Distinguishing neuroblastoma from Wilms tumor by computed tomography. J Comput Assist Tomogr 1985; 9: 889-893.

9. Fan R. Primary renal neuroblastoma--a clinical pathologic study of 8 cases. Am J Surg Pathol 2012; 36: $94-100$.

10. Dubois SG, London WB, Zhang Y, et al. Lung metastases in neuroblastoma at initial diagnosis: A report from the International Neuroblastoma Risk Group (INRG) project. Pediatr Blood Cancer 2008; 51: 589-592. 